\title{
Challenging clinical and organizational scenarios in cardiovascular diseases during the SARS-CoV-2 pandemic in Poland. Can we do better?
}

\author{
Wojciech Wojakowski ${ }^{1}$, Stanisław Bartuś ${ }^{2}$, Marek Grygier ${ }^{3}$ \\ ${ }^{1}$ Division of Cardiology and Structural Heart Diseases, Medical University of Silesia, Katowice, Poland \\ ${ }^{2} 2^{\text {nd }}$ Department of Cardiology, Jagiellonian University Medical Collage, Krakow, Poland \\ ${ }^{3} 1^{\text {st }}$ Department of Cardiology, Poznan University of Medical Sciences, Poznan, Poland
}

Adv Interv Cardiol 2020; 16, 2 (60): 121-122

DOI: https://doi.org/10.5114/aic.2020.95734

During the current SARS-Cov-2 pandemic, the management of patients with cardiovascular disease (CVD) should be focused not only on the improvement of outcomes but also the safety of both medical staff and patients, as well as limited healthcare resources and high demand for intensive care beds. Based on the previous experience from China, Italy, as well as other countries, all cardiology societies have issued guidelines for medical professionals to guide them through this challenging period. The Association of Cardiovascular Interventions of the Polish Cardiac Society (AISN PTK) on March 19, 2020, at the very beginning of the epidemic in Poland, published guidelines for the treatment of patients with acute coronary syndromes (ACS) in this setting. In addition, AISN PTK initiated numerous educational initiatives for healthcare professionals and information for patients disseminated on TV, radio, and social media.

What clinical and organizational issues are we facing when taking care of patients with ACS and high risk chronic coronary syndromes during the SARS-CoV-2 pandemic? Two interesting articles published in the current issue of Advances in Interventional Cardiology address these problems.

Broadly there are two categories of challenges. First, the clinical scenarios differ from what we are used to diagnose and treat. The presentation of ACS is different, with more patients presenting with symptoms suggestive of ACS. Registries show that a substantial number of patients with ST-segment elevation have no obstructive coronary disease. The increase of cardiac troponins is widespread and can represent myocardial inflammation rather than ischemia in a large percentage of cases. This can be related to diffuse inflammatory reaction involving the heart and is consistent with myocarditis, often with decompensated heart failure with high in-hospital mortality [1, 2]. The article of Bujak et al. describes the acute presentation of a patient with chest pain and ST-segment elevation, who was found to have no obstructive coronary lesions and proved to have COVID19 [3]. Therefore a low threshold for alternative diagnosis has to be taken. Also, given the high incidence of myocarditis mimicking ST-segment elevation acute myocardial infarction (MI), one has to be careful with advocating thrombolytic therapy instead of primary percutaneous coronary intervention $(\mathrm{PCl})$.

Secondly, there are profound changes in the way we diagnose and treat the patients because a balance has to be found to achieve the optimal therapeutic effects and, at the same time, diminish the risk of infection and overcome the organizational problems.

$\mathrm{PCl}$ saves lives and reduces morbidity in patients with ACS. In chronic coronary syndromes, $\mathrm{PCl}$ can reduce the angina burden and improve survival in patients with prognostically significant lesions such as unprotected left main, proximal left anterior descending artery, or single patent vessel stenosis. Therefore the dramatic reduction of $\mathrm{PCl}$ volumes during the SARS-CoV-2 pandemic may have a profound adverse impact on cardiovascular mortality and morbidity for many years to come. Every effort should be made to provide timely $\mathrm{PCl}$ for all patients in need. Reduction in $\mathrm{PCl}$ volumes can be system-related (closure of cathlabs due to administrative reasons, shortage of personal protection devices, focus on the treatment of COVID19-positive patients) as well as patient-related (fear of infection, anxiety, lack of family support, lack of information, disinformation spread through social

Corresponding author:

Stanisław Bartuś, $2^{\text {nd }}$ Department of Cardiology, Jagiellonian University Medical Collage, Krakow, Poland, e-mail: mbbartus@cyfronet.krakow.pl Received: 16.05 .2020 , accepted: 18.05 .2020 
media). In this aspect, the possibility of same-day discharge (SDD) PCI may alleviate many of these issues and likely reduce the risk of infection related to shorter hospitalization time. Such an approach can reduce the burden on the healthcare providers and medical staff, who during the night shifts will be available to focus on the care of other severely ill patients. SDD PCI has a proven safety record in all-comer populations of patients $[4,5]$. Nonetheless, patient selection is essential. Factors that might preclude safe SDD are severely reduced left ventricle ejection, chronic kidney disease, frailty, chronic obstructive pulmonary disease, and high bleeding risk.

SDD PCl in Poland is used infrequently, mainly due to the lack of specific reimbursement coding. The SARSCoV-2 pandemic can hopefully change it. The paper of Ciszewski is an excellent example of safely planned and conducted SDD. Importantly, all the rules of triage were followed (focused history taking, blood assay), the patient was separated from others, and the dedicated staff was responsible for him [6]. Such a strategy can relieve patients' anxiety, reduce the risk of infection and burden on hospital staff, and should be adopted in other cathlabs in Poland. It is very important not to miss the opportunity of early reperfusion in ST-segment elevation $\mathrm{MI}$, and we need to advocate for healthcare changes to stop the worrisome trend of declining $\mathrm{PCl}$ usage in Poland during the last years [7].

\section{Conflict of interest}

The authors declare no conflict of interest.

\section{References}

1. Wu Z, McGoogan JM. Characteristics of and important lessons from the coronavirus disease 2019 (COVID-19) outbreak in China: summary of a report of 72314 cases from the Chinese Center for Disease Control and Prevention. JAMA 2020; 323: 1239-42.

2. Fried JA, Ramasubbu K, Bhatt R, et al. The variety of cardiovascular presentations of COVID-19. Circulation 2020; 141: 1930-6.

3. Bujak K, Kazik A, Wróbel M, et al. COVID-19 mimicking ST-elevation myocardial infarction. Adv Interv Cardiol 2020; 16: 213-5.

4. Amin AH, Alqahtani F, Aljohani S, et al. The feasibility and safety of same day discharge for all comers after elective percutaneous coronary interventions. Cardiovasc Revasc Med 2019 October 23. doi: 10.1016/j.carrev.2019.09.024.

5. Taxiarchi P, Kontopantelis E, Martin GP, et al. Same-day discharge after elective percutaneous coronary intervention: insights from the British Cardiovascular Intervention Society. JACC Cardiovasc Interv 2019; 12: 1479-94.

6. Ciszewski A. Will the COVID19 pandemics acceralate the implementation of a single day coronary angioplasty in Poland? Adv Interv Cardiol 2020; 16: 184-6.

7. Dudek D, Siudak Z, Grygier M, et al. Interventional cardiology in Poland in 2019. Summary report of the Association of Cardiovascular Interventions of the Polish Cardiac Society (AISN PTK) and Jagiellonian University Medical College. Adv Interv Cardiol 2020; 16: 123-6. 